\title{
Numerical Stability for Some Equations of Gas Dynamics
}

\author{
By A. Y. Le Roux
}

\begin{abstract}
The isentropic gas dynamics equations in Eulerian coordinates are expressed by means of the density $\rho$ and the momentum $q=\rho u$, instead of the velocity $u$, in order to get domains bounded and invariant in the $(\rho, q)$-plane, for a wide class of pressure laws $p(\rho)$ and in the monodimensional case. A numerical scheme of the transport-projection type is proposed, which builds an approximate solution valued in such a domain. Since the characteristic speeds are bounded in this set, the stability condition is easily fulfilled and then estimates in the $L^{\infty}$-norm are derived at any time step. Similar results are extended to the model involving friction and topographical terms, and for a simplified model of supersonic flows. The nonapplication of this study to the gas dynamics in Lagrangian coordinates is shown.
\end{abstract}

1. Introduction. The isentropic gas dynamics equations in Eulerian coordinates may be written

$$
\begin{gathered}
\frac{\partial \rho}{\partial t}+\frac{\partial}{\partial x}(\rho u)=0, \quad(\rho=\text { density }) \\
\frac{\partial u}{\partial t}+\frac{\partial}{\partial x}\left(\frac{u^{2}}{2}\right)+\frac{1}{\rho} \frac{\partial}{\partial x}[p(\rho)]=0, \quad(u=\text { velocity })
\end{gathered}
$$

for $(x, t) \in] 0,1[\times] 0,+\infty[$. By using the momentum

$$
q=\rho u,
$$

we get the system

$$
\begin{gathered}
\frac{\partial \rho}{\partial t}+\frac{\partial q}{\partial x}=0 \\
\frac{\partial q}{\partial t}+\frac{\partial}{\partial x}\left[\frac{q^{2}}{\rho}+p(\rho)\right]=0 .
\end{gathered}
$$

Both models are nonlinear hyperbolic systems for $\rho>0$, when the pressure $p$ is an increasing function in $C^{2}(] 0,+\infty[)$ of the density $\rho$. We also suppose that $\rho(0)$ is zero and

$$
\forall \rho>0, \quad \rho p^{\prime \prime}(\rho)+2 p^{\prime}(\rho) \geqslant 0 .
$$

From this last condition, both systems are genuinely nonlinear (see [4], [5]). Note that the hypotheses include all functions $p(\rho)=\rho^{\gamma}$ with $\gamma>0$. Initial data $\rho_{0}$ (nonnegative) and $u_{0}$ are prescribed in $L^{\infty}(] 0,1[)$. Moreover $u$ and thus $q$ by (1.3) are assumed to be zero for any $t>0$ at $x=0$ and at $x=1$.

Received June 30, 1980; revised February 17, 1981.

1980 Mathematics Subject Classification. Primary 65M10, 35L65.

(C) 1981 American Mathematical Society 0025-5718/81/0000-0154/\$04.50 
Regular solutions are obviously the same for both systems. It is not true for the solutions with shocks (i.e. discontinuous solutions) which may occur though the model is claimed to be isentropic. It is well known that the solutions with shocks given by the $(\rho, q)$-model are the correct ones. Moreover, the $(\rho, q)$-model has domains invariant, bounded, and convex in the $(\rho, q)$-plane. This property is not always satisfied by the $(\rho, u)$ model, particularly for the functions $p$ used in practice. We shall thus mainly consider the $(\rho, q)$-system, for which the Riemann problem is solved in Section 2.

A numerical explicit scheme is proposed in Section 3. It consists of two steps, the one a transport of the exact solution computed from constant piecewise initial data during a time interval of length $\Delta t$, the other an orthogonal projection in $L^{2}([0,1[)$ on the same class of constant piecewise functions. The stability in the $L^{\infty}$-norm is deduced from the properties of convexity and boundedness of the invariant sets for the $(\rho, q)$-model, provided that a stability condition (of Courant-Friedrichs-Lewy) is satisfied. The scheme we get this way is a variant of the Godunov scheme; see [3]. The same results are stated in Section 4 for the Lax-Friedrichs scheme and for the Glimm scheme.

A generalization of these schemes to a model including friction and topographical terms is studied in Section 5 . We prove that the $L^{\infty}$-norm is growing slowly enough, so that computation is possible up to any time $t$, with the stability condition always satisfied.

In Section 6 we try to apply the same technique to the simplified supersonic flow equation

$$
\frac{\partial^{2} w}{\partial t^{2}}-\frac{\partial}{\partial x}\left[f\left(\frac{\partial w}{\partial x}\right)\right]=0
$$

with $f \in C^{2}(\mathbf{R})$, decreasing on $]-\infty, 0[$ and increasing on $] 0,+\infty[$.

This problem is hyperbolic only for $u=\partial w / \partial x$ positive. We show that nonexistence may occur near the sonic value $u=0$, for (1.7). We then propose a new formulation of this problem by using the variables $\rho=u$ and a momentum $q=u \partial w / \partial t$, for which existence for the Riemann problem is proved for $\rho>0$. The results of Sections 3, 4, and 5 are suitable here, and with hypotheses similar to (1.6). Note that the variable $q$ has no well-known physical meaning.

Similar results were stated in [10] for (1.7) with $f$ increasing on $\mathbf{R}, f(0)=0$ and $|f|$ convex; this is a nonlinear model for strings which are not hardly stretched. Bounded and convex invariant sets exist in the phase plane, without any transformation on the functions $u=\partial w / \partial t$ and $v=\partial w / \partial x$. However, these results are not applicable for gas dynamics in Lagrangian coordinates, which we may write

$$
\frac{\partial u}{\partial t}+\frac{\partial}{\partial x} p(v)=0, \quad \frac{\partial v}{\partial t}=\frac{\partial u}{\partial x} .
$$

Here $v\left(=\rho^{-1}\right)$ is the specific volume, $u$ is the velocity and $p$ is the pressure. The system (1.8) has convex invariant domains in the $(v, u)$-plane, for $p(v)=v^{-\gamma}$ with $1<\gamma<3$, but they are unbounded. By using a momentum $q=u v$, a new conservative form of (1.8) appears, for which invariant sets are bounded and convex in the $(v, q)$-plane. Unfortunately, the characteristic speeds may be infinite in these domains, and then the stability condition will never be satisfied for $v$ close to zero. Thus, the transformation $q=u v$ has no interest for (1.8). 
2. The Riemann Problem. This problem will be used for the construction of numerical schemes. It consists in finding the solution $(\rho, q)$ of $(1.4),(1.5)$ with initial conditions on $\mathbf{R}$, which are constant on $]-\infty, 0[$ and on $] 0,+\infty[$, and given by

$$
\left(\rho_{0}(x), q_{0}(x)\right)= \begin{cases}\left(\rho_{-}, \rho_{-} u_{-}\right) & \text {for } x<0 \\ \left(\rho_{+}, \rho_{+} u_{+}\right) & \text {for } x>0\end{cases}
$$

with $\rho_{-} \geqslant 0, \rho_{+} \geqslant 0$.

The solution to this problem is given by two waves of different speeds, and the value in between is a constant. Such a wave may be a rarefaction or a shock wave. We first analyze a rarefaction wave, corresponding to a couple $(\rho, q)$ of functions which are continuous and monotone with respect to $w$ at any time level $t$. They are given by the Riemann invariants, and we obtain them easily by writing $q$ as a function of $\rho$ (i.e., by eliminating $x$ ) in (1.4) and (1.5) we get a first-order differential equation, giving two solutions that we combine with the initial data (2.1) to get

$$
\left\{\begin{array}{l}
q=\rho\left(u_{+}+P_{1}(\rho)-P_{1}\left(\rho_{+}\right)\right) \\
q=\rho\left(u_{-}-P_{1}(\rho)+P_{1}\left(\rho_{-}\right)\right)
\end{array}\right.
$$

with $P_{1}$ defined by

$$
P_{1}(\rho)=\int_{1}^{\rho} \frac{1}{y} \sqrt{p^{\prime}(y)} d y
$$

Each invariant in (2.2) describes a wave, the first one is called of the first kind, the second one of the second kind. A first kind wave is always faster than a second kind wave, and the sign of their speeds is generally different (for subsonic stream). In (2.2), $q$ is a convex function of $\rho$ for the plus sign (first kind), and a concave one for the minus sign (second kind). From the scalar theory, such rarefaction waves only occur if $\rho$ is increasing on a first kind wave, decreasing on a second kind wave.

A shock wave corresponds to a discontinuous solution of (1.4) and (1.5) and is ruled by the well-known Rankine-Hugoniot jump condition. A discontinuity curve of equation $x=x(t)$ must satisfy

$$
x^{\prime}(t)=\frac{q_{1}-q_{2}}{\rho_{1}-\rho_{2}}=\frac{1}{q_{1}-q_{2}}\left\{\frac{q_{1}^{2}}{\rho_{1}}+p\left(\rho_{1}\right)-\frac{q_{2}^{2}}{\rho_{2}}-p\left(\rho_{2}\right)\right\},
$$

where $\left(\rho_{1}, q_{1}\right)$ and $\left(\rho_{2}, q_{2}\right)$ are the values on each side of the jump. We thus obtain $q_{2}$ as a function of $\rho_{2}$, with $\left(\rho_{1}, q_{1}\right)$ fixed, namely

$$
q_{2}=\frac{q_{1}}{\rho_{1}} \rho_{2} \pm\left\{\frac{\rho_{2}}{\rho_{1}}\left(\rho_{2}-\rho_{1}\right)\left(p\left(\rho_{2}\right)-p\left(\rho_{1}\right)\right)\right\}^{1 / 2} .
$$

As before the plus sign corresponds to a shock wave of the first kind, the minus sign to a second kind wave. Note that the jump condition for (1.1) and (1.2) gives other shock waves, the speeds of which are different from those given by (2.4). Moreover, the shock waves must satisfy an entropy condition, in order to be physically meaningful. This is deduced from the scalar theory (see [5], [6]). Since 
the system is genuinely nonlinear from (1.6), this condition is

$$
\begin{cases}\rho(x-0, t) \geqslant \rho(x+0, t) & \text { for the first kind, } \\ \rho(x-0, t) \leqslant \rho(x+0, t) & \text { for the second kind. }\end{cases}
$$

The Riemann problem is solved by drawing in the $(\rho, q)$-plane the two curves

$$
S_{+}\left(\rho_{+}, q_{+}\right)
$$

$$
=\left\{(\rho, q) ; q=\left\{\begin{array}{ll}
p\left[u_{+}+P_{1}(\rho)-P_{1}\left(\rho_{+}\right)\right] & \text {if } 0<\rho<\rho \\
q_{+}+\sqrt{\frac{\rho_{+}}{\rho}\left(\rho-\rho_{+}\right)\left(p(\rho)-p\left(\rho_{+}\right)\right)} & \text {if } \rho>\rho_{+}
\end{array}\right\},\right.
$$

$$
S_{-}\left(\rho_{-}, q_{-}\right)
$$

$$
=\left\{(\rho, q) ; q=\left\{\begin{array}{ll}
\rho\left[u_{-}-P_{1}(\rho)+P_{1}\left(\rho_{-}\right)\right] & \text {if } 0<\rho<\rho_{-} \\
q_{-}-\sqrt{\frac{\rho_{-}}{\rho}\left(\rho-\rho_{-}\right)\left(p(\rho)-p\left(\rho_{-}\right)\right)} & \text {if } \rho>\rho_{-}
\end{array}\right\},\right.
$$

in which $q_{+}=\rho_{+} u_{+}, q=\rho_{-} u_{-}$. The curve (2.7) describes the states which can be connected to $\left(\rho_{+}, q_{+}\right)$by a first kind wave, the curve (2.6) the states connected to $\left(\rho_{-}, q_{-}\right)$by a second kind wave. Thus the constant state of the solution between these two waves is given by the intersection $(\tilde{\rho}, \tilde{q})$ of these two curves, such that $\tilde{\rho}>0$ for $S_{-}\left(\rho_{-}, q_{-}\right)$lying below $S_{+}\left(\rho_{+}, q_{+}\right)$for $\rho>\tilde{\rho}$ (i.e., take another $(\tilde{\rho}, \tilde{q})$ than the trivial one if possible). We shall also need the value of the solution on the line $x=0$; it is a constant $\left(\rho_{s}, q_{s}\right)$ given by the formula

$$
\left(\rho_{s}, q_{s}\right)=\left[\begin{array}{c}
\left(\rho_{+}, q_{+}\right) \quad \text { if } \lambda_{+}\left(\rho_{+}, q_{+}\right)<0, \tilde{\rho}<\rho_{+}, \text {or if } \tilde{q}<q_{+}, \tilde{\rho}>\rho_{+}, \\
\left(\rho_{-}, q_{-}\right) \text {if } \lambda_{-}\left(\rho_{-}, q_{-}\right) \geqslant 0, \tilde{\rho}<\rho_{-}, \text {or if } \tilde{q}>q_{-}, \tilde{\rho}>\rho_{-}, \\
s_{+}\left(\rho_{+}, q_{+}\right) \cap\left\{\lambda_{+}(\rho, q)=0\right\} \\
\quad \text { if } \lambda_{+}(\tilde{\rho}, \tilde{q})<0<\lambda_{+}\left(\rho_{+}, q_{+}\right), \tilde{\rho}<\rho_{+}, \\
S_{-}\left(\rho_{-}, q_{-}\right) \cap\left\{\lambda_{-}(\rho, q)=0\right\} \\
\quad \text { if } \lambda_{-}(\tilde{\rho}, \tilde{q})>0>\lambda_{-}\left(\rho_{-}, q_{-}\right), \tilde{\rho}<\rho_{-}, \\
(\tilde{\rho}, \tilde{q}) \quad \text { in other cases, }
\end{array}\right.
$$

with $\lambda_{+}(\rho, q)$ and $\lambda_{-}(\rho, q)$ given by

$$
\lambda_{+}(\rho, q)=\frac{q}{\rho}+\sqrt{p^{\prime}(\rho)}, \quad \lambda_{-}(\rho, q)=\frac{q}{\rho}-\sqrt{p^{\prime}(\rho)} .
$$

These values $\lambda_{ \pm}(\rho, q)$ correspond to the speed of each kind of wave. By writing $u=q / \rho$ and $c=\sqrt{p^{\prime}(\rho)}$ (= sound speed), we get $\lambda_{ \pm}=u \pm c$. They are of opposite sign for $|u|<c$ (i.e., for subsonic stream) and then $(\tilde{\rho}, \tilde{q})$ is often selected in (2.9).

We have the following

THEOREM 1. The Riemann problem (1.4), (1.5), (2.1) has a unique solution, which is valued in the bounded convex set of the $(\rho, q)$-plane

$$
K\left(R_{0}\right)=\left\{(\rho, q) ; \rho \geqslant 0,|q|+\rho\left(P_{1}(\rho)-R_{0}\right)<0\right\},
$$

for any real positive number $R_{0}$ such that

$$
\operatorname{Max}\left\{\left|u_{+}\right|+P_{1}\left(\rho_{+}\right),\left|u_{-}\right|+P_{1}\left(\rho_{-}\right)\right\}<R_{0}<\lim _{\rho \rightarrow \infty} P_{1}(\rho) .
$$


Moreover, the solution is equal to $\left(\rho_{s}, q_{s}\right)$, given by (2.9), for $x=0$ and satisfies

$$
(\rho(x, t), q(x, t))= \begin{cases}\left(\rho_{-}, q_{-}\right) & \text {for } x<-\lambda\left(R_{0}\right) t, \\ \left(\rho_{+}, q_{+}\right) & \text {for } x>\lambda\left(R_{0}\right) t,\end{cases}
$$

with

$$
\lambda\left(R_{0}\right)=\operatorname{Sup}_{(\rho, q) \in K\left(R_{0}\right)}\left\{\frac{|q|}{\rho}+\sqrt{p^{\prime}(\rho)}\right\} .
$$

Proof. The set $K\left(R_{0}\right)$ is obviously convex from (1.6) and bounded from (2.12) since $R_{0}$ is strictly less than the upper limit of $P_{1}$ (which is often infinite), and since we get for $\rho<1$ by the Cauchy-Schwarz inequality

$$
\rho\left|P_{1}(\rho)\right| \leqslant \rho\left(\int_{\rho}^{1} \frac{d y}{y^{2}}\right)^{1 / 2}\left(\int_{\rho}^{1} p^{\prime}(y) d y\right)^{1 / 2} \leqslant \sqrt{\rho p(1)},
$$

which implies that $\rho\left|P_{1}(\rho)\right| \rightarrow 0$ if $\rho \rightarrow 0$. This proves the theorem as long as the existence of a solution is concerned since from (2.12) the intersection giving $\left(\rho_{s}, q_{s}\right)$ is never empty. Uniqueness is deduced from a result of T. P. Liu (see [11]), since the entropy condition (2.6) is fulfilled.

Obviously, $K\left(R_{0}\right)$ contained the initial values from (2.12). Since its boundaries are Riemann invariants, from (2.2), the rarefaction waves are valued in $K\left(R_{0}\right)$. Now we have to show that it is also true for a shock wave. For a shock wave of the first kind, we have an inequality between the shock curve $q_{2}\left(\rho_{2}\right)$ given by $(2.5)$ and the Riemann invariant $q\left(\rho_{2}\right)$ starting from the same point $\left(\rho_{1}, q_{1}\right)$ with $\rho_{2}>\rho_{1}$. Namely, by the Cauchy-Schwarz inequality, we have

$$
\begin{aligned}
q\left(\rho_{2}\right) & =\frac{q_{1}}{\rho_{1}} \rho_{2}+\int_{\rho_{1}}^{\rho_{2}} \frac{1}{y} \sqrt{p^{\prime}(y)} d y \rho_{2} \\
& \leqslant \frac{q_{1}}{\rho_{1}} \rho_{2}+\left(\int_{\rho_{1}}^{\rho_{2}} \frac{d y}{y^{2}}\right)^{1 / 2}\left(\int_{\rho_{1}}^{\rho_{2}} p^{\prime}(y) d y\right)^{1 / 2} \rho_{2}=q_{2}\left(\rho_{2}\right) .
\end{aligned}
$$

It follows that the values of a shock curve (2.5) always lie on the inside of the convexity of the Riemann invariant starting from the same point $\left(\rho_{1}, q_{1}\right)$, and then belong to $K\left(R_{0}\right)$. We have the same for a shock wave of the second kind.

Let us now show (2.13). This is obvious when only rarefaction waves occur. The speed of a first kind shock wave, given by (2.4), may be estimated as follows. We multiply (1.6) by $\left(\rho-\rho_{1}\right)$, with $\rho>\rho_{1}$, so as to get

$$
\left(\rho-\rho_{1}\right)\left(\rho p^{\prime \prime}(\rho)+p^{\prime}(\rho)\right)+\rho p^{\prime}(\rho)>\rho_{1} p^{\prime}(\rho) .
$$

Integration on $] \rho_{1}, \rho_{2}[$ yields

$$
\left(\rho_{2}-\rho_{1}\right) \rho_{2} p^{\prime}\left(\rho_{2}\right) \geqslant \rho_{1}\left[p\left(\rho_{2}\right)-p\left(\rho_{1}\right)\right],
$$

which gives

$$
\sqrt{p^{\prime}\left(\rho_{2}\right)} \geqslant \sqrt{\frac{\rho_{1}}{\rho_{2}} \frac{p\left(\rho_{2}\right)-p\left(\rho_{1}\right)}{\rho_{2}-\rho_{1}}} .
$$

When adding $q_{2} / \rho_{2}$, we get exactly

$$
\left|x^{\prime}(t)\right|<\left|\lambda_{+}\left(\rho_{2}, q_{2}\right)\right| \quad\left(<\lambda\left(R_{0}\right)\right),
$$


which means that a shock wave is slower than a characteristic line along which the value $\left(\rho_{2}, q_{2}\right)$ is travelling. The same arguments are suitable for a shock wave of the second kind. Since the solution was already shown to be given by (2.9) for $x=0$, the proof of Theorem 1 is complete.

The inequality (2.15) may not hold for the $(\rho, u)$-model (1.1),(1.2). For example, if $p(\rho)=\rho^{2}$ (i.e., the shallow water equations), the shock curves are lying on one side of the Riemann invariant for the $(\rho, u)$-model, and on the other side (the correct one) for the $(\rho, q)$-model, since the convexity of the Riemann invariant is changed by the transformation $q=\rho u$. This example was studied in [8] or [9].

3. Stability in the $L^{\infty}$-Norm for a Numerical Scheme. Let $I \in N ; h=1 / I$ is the space mesh size. We consider the space

$$
V_{h}=\left\{v \in L^{\infty}(] 0,1[), v=\text { Constant on } I_{i+1 / 2}=\right] i h,(i+1) h[,
$$

$$
0<i<I-1\}
$$

and the orthogonal projection on $V_{h}$, associated to the $L^{2}$-norm, which is nothing but the average on each $I_{i+1 / 2}$. We use it to approximate the initial data by putting

$$
\rho_{i+1 / 2}^{0}=\frac{1}{h} \int_{I_{i+1 / 2}} \rho_{0}(x) d x, \quad q_{i+1 / 2}^{0}=\frac{1}{h} \int_{I_{i+1 / 2}} \rho_{0}(x) u_{0}(x) d x,
$$

for $i=0, \ldots, I-1$.

The time mesh size is $\Delta t=r h$, where $r$ is a real positive number corresponding to the ratio of mesh sizes and to be used in a stability condition with the maximal speed of the waves. This ratio is a constant only when the $L^{\infty}$-norm of the solution is uniformly bounded since the speed of the waves is depending on the values of the solution. At time level $t_{n}=n \Delta t$ with $n \in \mathbf{N}$, we suppose we know the approximate solution $\left(\rho^{n}, q^{n}\right) \in V_{h} \times V_{h}$, the values of which are denoted by $\left(\rho_{i+1 / 2}^{n}, q_{i+1 / 2}^{n}\right)$ on $I_{i+1 / 2}$ for $i=0, \ldots, I-1$ with $\rho_{i+1 / 2}^{n}$ nonnegative.

We shall compute the approximate solution at time level $t_{n+1}$ by a two-step method. The first step uses the result of Section 2 to build the exact solution on the strip $] 0,1[\times] t_{n}, t_{n+1}[$, and the second step is an orthogonal projection of this solution on $V_{h} \times V_{h}$. Fortunately we only need the values given by (2.9) and thus this method gives an explicit scheme of order one of accuracy, which is a rather good performing scheme with a low damping. This looks like the natural generalization of the Godunov scheme or of the upwind scheme used for the scalar equation; see [3], [6], [7].

This scheme is as follows. First we compute for $i=1, \ldots, I-1$,

$$
\left(\tilde{\rho}_{i}^{n}, \tilde{q}_{i}^{n}\right) \in S_{+}\left(\rho_{i+1 / 2}^{n}, q_{i+1 / 2}^{n}\right) \cap S_{-}\left(\rho_{i-1 / 2}^{n}, q_{i-1 / 2}^{n}\right)
$$

such that $S_{-}$lies below $S_{+}$for $\rho>\tilde{\rho}_{i}^{n}$, as for $(\tilde{\rho}, \tilde{q})$ above. We take the line $\{q=0\}$ instead of $S_{+}$for $x=0$ or of $S_{-}$for $x=1$, at the boundaries. Namely, we get

$$
\begin{aligned}
\left(\rho_{0}^{n}, 0\right) \in S_{+}\left(\rho_{1 / 2}^{n}, q_{1 / 2}^{n}\right) \cap\{q=0\}, & q_{0}^{n}=0, \\
\left(\rho_{I}^{n}, 0\right) \in S_{-}\left(\rho_{I-1 / 2}^{n}, q_{I-1 / 2}^{n}\right) \cap\{q=0\}, & q_{I}^{n}=0,
\end{aligned}
$$

again with $S_{-}$below the line $\{q=0\}$ for $\rho>\rho_{I}^{n}$ or $S_{+}$above this line for $\rho>\rho_{0}^{n}$. The arguments for (3.5) and (3.6) come from the resolution of a "half Riemann 
problem" on $] 0,+\infty[\times] 0,+\infty[$ or on $]-\infty, 0[\times] 0,+\infty[$, which is performed as in Section 2. Note that the solution of such a problem has only one wave; of the first kind with a positive speed in the first case, of the second kind with a negative speed in the second case. Obviously Theorem 1 is also valid for such problems.

Then for $i=1, \ldots, I-1$, we compute the stationary values

$$
\left(\rho_{i}^{n}, q_{i}^{n}\right)=\left(\rho_{s}, q_{s}\right)
$$

given by (2.9) with

$$
\left(\rho_{+}, q_{+}\right)=\left(\rho_{i+1 / 2}^{n}, q_{i+1 / 2}^{n}\right), \quad\left(\rho_{-}, q_{-}\right)=\left(\rho_{i-1 / 2}^{n}, q_{i-1 / 2}^{n}\right), \quad(\rho, q)=\left(\tilde{\rho}_{i}^{n}, q_{i}^{n}\right) .
$$

The values given by (3.5), (3.6), and (3.7) are exactly the values of the solution on each segment $\{i h\} \times] t_{n}, t_{n+1}[$, provided that $\Delta t$ is small enough so that two waves cannot meet each other before $t_{n+1}$. This will be ensured by a stability condition of the Courant-Friedrichs-Lewy type which uses (2.13).

Now, for the second step, we compute the averages of $\rho$ and $q$ on each $I_{i+1 / 2}$ at time $t_{n+1}$. They are directly obtained by a Green's formula on (1.4) and (1.5). We get for $i=0, \ldots, I-1$,

$$
\begin{gathered}
\rho_{i+1 / 2}^{n+1}=\rho_{i+1 / 2}^{n}-r\left(q_{i+1}^{n}-q_{i}^{n}\right), \\
q_{i+1 / 2}^{n+1}=q_{i+1 / 2}^{n}-r\left[\frac{\left(q_{i+1}^{n}\right)^{2}}{\rho_{i+1}^{n}}+p\left(\rho_{i+1}^{n}\right)-\frac{\left(q_{i}^{n}\right)^{2}}{\rho_{i}^{n}}-p\left(\rho_{i}^{n}\right)\right] .
\end{gathered}
$$

The first step corresponds to a transport phase given by (3.4), (3.5), (3.6), and (3.7), and the second step to a projection phase, given by (3.8) and (3.9). We have built a "transport-projection method", for which we have the following

TheOREM 2. For $\rho_{0} \in L^{\infty}(] 0,1[)$ nonnegative and $u_{0} \in L^{\infty}(] 0,1[)$ such that

$$
R_{0}=\operatorname{Sup}_{x \in] 0,1[}\left\{\left|u_{0}(x)\right|+P_{1}\left(\rho_{0}(x)\right)\right\}<\lim _{\rho \rightarrow \infty} P_{1}(\rho) \text {, }
$$

and provided the stability condition

$$
r \lambda\left(R_{0}\right) \leqslant \frac{1}{2}
$$

holds with $\lambda\left(R_{0}\right)$ given by (2.14), the scheme (3.4) (3.5) (3.6) (3.7) (3.8) (3.9) works up to any time $t_{n}, n \in \mathbf{N}$. Moreover, the values of the approximate solution always lie in the bounded convex set $K\left(R_{0}\right)$ defined in (2.11).

Proof. From Theorem 1 and since (2.12) is obviously satisfied, the existence of a solution on each strip $] 0,1[\times] t_{n}, t_{n+1}$ [ computed from given values in $V_{h} \times V_{h}$ is ensured. The Riemann problems have no interaction between themselves before time $t_{n+1}$ by (2.13) since (3.11) holds. All values of the solutions of these Riemann problems belong to $K\left(R_{0}\right)$ which is a bounded convex set. Thus their projections on $V_{h} \times V_{h}$ given by (3.8) and (3.9) also belong to $K\left(R_{0}\right)$. Theorem 2 is proved.

This theorem states that $K\left(R_{0}\right)$ is a numerically invariant set for (1.4) and (1.5). The property to have such convex invariant sets which are also bounded is inherent to the $(\rho, q)$-model. On the other hand, this is not true for the $(\rho, u)$-model for $p(\rho)=\rho^{\gamma}$ with $0<\gamma<3$. This is shown in [8], [9] for the shallow water equations (i.e., (1.1), (1.2) with $p(\rho)=\rho^{2}$ ). Boundedness is true for $\gamma>3$; see [1].

Note that (3.11) is stronger than the Courant-Friedrichs-Lewy stability condition since it involves the factor $\frac{1}{2}$ instead of 1 . This comes from the fact that we have 
not studied the interaction of two waves of different kind; thus we have to project before they meet. Otherwise existence would not be proved on the whole strip ] $0,1[\times] t_{n}, t_{n+1}[$. However, the values given by (3.7) may be also valid for the case $r \lambda\left(R_{0}\right) \leqslant 1$, and the conclusions of Theorem 2 still seem to hold.

4. The Lax-Friedrichs and Glimm Schemes. Theorem 2 may be extended to the Lax-Friedrichs scheme and to the Glimm scheme rather easily since they can be presented as two-step methods: transport and projection for the first one, transport and random interpolation for the second.

Let $I$ be an even number. For $h=1 / I$, we consider the spaces

$$
\begin{array}{r}
V_{h}^{\nu}=\left\{v L^{\infty}(] 0,1[), v=\text { constant on }\right](i-1) h,(i+1) h[\cap] 0,1[ \\
\text { for } i+\nu \text { odd }\},
\end{array}
$$

for $\nu=0$ and $\nu=1$. The initial data are projected on $V_{h}^{0}$ by putting

$$
\rho_{i}^{0}=\frac{1}{2 h} \int_{(i-1) h}^{(i+1) h} \rho_{0}(x) d x, \quad q_{i}^{0} \int_{(i-1) h}^{(i+1) h} \rho_{0}(x) u_{0}(x) d x
$$

for $i=1,3, \ldots, I-1$.

As before we introduce the sequence $t_{n}=n r h$ and suppose we know all the $\left(\rho_{i}^{n}, q_{i}^{n}\right)$ for $n$ even and $i=1,3, \ldots, I-1$. We solve the problem (1.4), (1.5) on the strip $] 0,1[\times] t_{n}, t_{n+1}\left[\right.$, starting from these values at time $t_{n}$. This solution is constant on each triangle

$$
T_{i}^{n}=\left\{(x, t) ;|x-i h|+\frac{1}{r}\left|t-t_{n}\right| \leqslant h\right\},
$$

if $r h$ is small enough, which we shall ensure by the stability condition of CourantFriedrichs-Lewy. Then we project this solution on $V_{h}^{1}$ at time $t_{n+1}$ by averaging on each $](i-1) h,(i+1) h[\cap] 0,1[$ for $i$ even. Now we solve once again (1.4) (1.5) on the strip $] 0,1[\times] t_{n+1}, t_{n+2}\left[\right.$, and then project on $V_{h}^{0}$, and so on. Since the solution on each strip is constant on each $T_{j}^{n}$ for $j+n$ odd, the averages on $](i-1) h$, $(i+1) h$ [ at time $t_{n+1}$, that we denote by $\left(\rho_{i}^{n+1}, q_{i}^{n+1}\right)$, are easily computed by a Green's formula on the set $](i-1) h,(i+1) h[\times] t_{n}, t_{n+1}[$. This gives the wellknown Lax-Friedrichs scheme, for $i+n$ even,

$$
\begin{gathered}
\rho_{i}^{n+1}=\frac{1}{2}\left(\rho_{i+1}^{n}+\rho_{i-1}^{n}\right)-\frac{r}{2}\left(q_{i+1}^{n}-q_{i-1}^{n}\right), \\
q_{i}^{n+1}=\frac{1}{2}\left(q_{i+1}^{n}+q_{i-1}^{n}\right)-\frac{r}{2}\left\{\frac{\left(q_{i+1}^{n}\right)^{2}}{\rho_{i+1}^{n}}+p\left(\rho_{i+1}^{n}\right)-\frac{\left(q_{i-1}^{n}\right)^{2}}{\rho_{i-1}^{n}}-p\left(\rho_{i-1}^{n}\right)\right\} .
\end{gathered}
$$

The boundary values are taken in account only for $n$ even, that is

$$
\rho_{0}^{n+1}=\frac{1}{2}\left(\rho_{1}^{n}-r q_{1}^{n}\right), \quad q_{0}^{n+1}=\frac{1}{2}\left(q_{1}^{n}-r\left(\frac{\left(q_{1}^{n}\right)^{2}}{\rho_{1}^{n}}+p\left(\rho_{1}^{n}\right)-p\left(\tilde{\rho}_{0}^{n}\right)\right)\right),
$$

at $x=0$, in which

$$
\left(\tilde{\rho}_{0}^{n}, 0\right)=S_{+}\left(\rho_{1}^{n}, q_{1}^{n}\right) \cap\{q=0\}
$$


as in (3.5), with $S_{+}$above $\{q=0\}$ for $\rho>\rho_{0}^{n}$. By the same arguments at $x=1$,

$$
\begin{aligned}
\rho_{I}^{n+1}= & \frac{1}{2}\left(\rho_{I-1}^{n}-r q_{I-1}^{n}\right), \\
q_{I}^{n+1}= & \frac{1}{2}\left(q_{I-1}^{n}-r\left(\frac{\left(q_{I-1}^{n}\right)^{2}}{\rho_{I-1}^{n}}+p\left(\rho_{I-1}^{n}\right)-p\left(\tilde{\rho}_{I}^{n}\right)\right)\right), \\
& \left(\tilde{\rho}_{I}^{n}, 0\right) \in S_{-}\left(\rho_{I-1}^{n}, q_{I-1}^{n}\right) \cap\{q=0\} .
\end{aligned}
$$

We have the following result:

TheOREM 3. For $\rho_{0} \in L^{\infty}\left(\left[0,1[)\right.\right.$ nonnegative and $u_{0} \in L^{\infty}\left(\left[0,1[)\right.\right.$, such that $R_{0}$ satisfying (3.10) exists, and provided that the C.F.L. stability condition

$$
r \lambda_{0}\left(R_{0}\right) \leqslant 1
$$

holds, the Lax-Friedrichs scheme (4.3), (4.4) with (4.5), (4.6), (4.7), and (4.8) for the boundaries, works up to any time $t_{n}, n \in \mathbf{N}$. Moreover, the values of the approximate solution always belong to the bounded and convex set $K\left(R_{0}\right)$.

The same arguments as above are used to prove this theorem since from (2.13) the solution is constant on each $T_{j}^{n}, j+n$ odd, in each strip if (4.9) holds. Thus we do not give any details of this proof here. Note only that the right C.F.L. condition is accepted here. For each interval inside ]0, $1[$ has a length $2 h$, it is not less restrictive than (3.11) when $V_{h}$ is used. The natural damping for this scheme is rather important and increases as $r$ decreases, which was not the case for the scheme (3.8) (3.9).

The Glimm scheme uses the same spaces $V_{h}^{0}$ and $V_{h}^{1}$ as in the Lax-Friedrichs scheme, but a random interpolation is performed instead of an $L^{2}$-projection at each time $t_{n}$. This is done as follows. A sequence of random numbers

$$
\alpha_{0}, \alpha_{1}, \ldots, \alpha_{n}, \ldots \in A=\prod_{i=0}^{\infty}[0,1]
$$

is given, and we take

$$
\left(\rho_{i}^{n+1}, q_{i}^{n+1}\right)=\left(\rho^{n}\left(x_{n}, t_{n+1}\right), q^{n}\left(x_{n}, t_{n+1}\right)\right)
$$

for $x_{n}=\left(i+1-2 \alpha_{n}\right) h$ and $i+n$ even. In (4.10) $\left(\rho^{n}, q^{n}\right)$ is the solution of (1.4), (1.5) computed in the strip $] 0,1[\times] t_{n}, t_{n+1}\left[\right.$, starting from the data $\left(\rho_{j}^{n}, q_{j}^{n}\right)$ at time $t_{n}$, or given by (4.2) for $n=0$. We have the same conclusions as in Theorem 3, with the same C.F.L. condition. The convexity of $K\left(R_{0}\right)$ is not explicitly needed here, but seems to be a necessary condition for invariance and thus is required implicitly.

Note also that the Lax-Friedrichs scheme and the scheme given in Section 3 have the property of the conservation of the mass, that is, respectively,

$$
\sum_{i+n \text { even }} \rho_{i}^{n+1} h_{i}=\sum_{j+n \text { odd }} \rho_{j}^{n} h_{j}=\cdots=\sum_{k \text { odd }} \rho_{k}^{0} h_{k}=\int_{0}^{1} \rho_{0}(x) d x,
$$

where $h_{i}$ is the length of $](i-1) h,(i+1) h[\cap] 0,1[$, and

$$
\begin{aligned}
\sum_{0<i<I-1} \rho_{i+1 / 2}^{n+1} h & =\sum_{0<i<I-1} \rho_{i+1 / 2}^{n} h=\cdots=\sum_{0<i<I-1} \rho_{i+1 / 2} h \\
& =\int_{0}^{1} \rho_{0}(x) d x .
\end{aligned}
$$


This means also the conservation of the $L^{1}$-norm of $\rho$, since $\rho$ is nonnegative, for we have the invariance of $K\left(R_{0}\right) \subset\{(\rho, q), \rho \geqslant 0\}$. The conservation of the mass is only approximately satisfied when one uses the Glimm scheme. An analogue of the Glimm scheme may also be built by using the space $V_{h}$, that is by taking a random interpolation on each $I_{i+1 / 2}$ at time $t_{n}$ instead of a projection. The result of Theorem 2 would hold for it.

5. Introduction of a Second Member. We consider now the system

$$
\begin{gathered}
\frac{\partial \rho}{\partial t}+\frac{\partial q}{\partial x}=0 \\
\frac{\partial q}{\partial t}+\frac{\partial}{\partial x}\left(\frac{q^{2}}{\rho}+p(\rho)\right)+b(\rho, q) q+a(x) \rho=0
\end{gathered}
$$

with

$$
a \in C^{0}([0,1]), \quad b \in C^{0}(] 0, \infty[\times \mathbf{R})
$$

and satisfying

$$
\forall(\rho, q) \in K\left(R_{0}\right), \quad 0 \leqslant b(\rho, q)|q| \leqslant k_{0}\left(R_{0}\right),
$$

for any $R_{0}>0$ and for some constant $k_{0}\left(R_{0}\right)$. The function $a$ corresponds physically to the slope of the topography and $b$ to a friction term. We find usually $b(\rho, q)=|q| / \rho$, satisfying (5.4) with

$$
k_{0}\left(R_{0}\right)=\left(R_{0} \sqrt{\rho_{*}}+\sqrt{p(1)}\right)^{2}, \quad \rho_{*}=P_{1}^{-1}\left(R_{0}\right) .
$$

The same schemes as above are studied now. Since the $L^{\infty}$-norm of the solution may grow, we have to work with a sequence $r_{n}$ defined by

$$
r_{n}=\left(t_{n+1}-t_{n}\right) / h \text {. }
$$

The sequence $r_{n}$ may decrease to spare the stability of the scheme. We shall show that $r_{n}$ is decreasing slowly enough to have the sequence $t_{n}$ divergent. Thus any time $T \in] 0,+\infty[$ can be reached. From (5.4) $b$ is nonnegative and thus works as a damping term, preserving $K\left(R_{0}\right)$. The increasing of the norm can only come from the term $a$.

For the scheme studied in Section 3, the only difference from above is to replace (3.9) with

$$
\left[1+r_{n} h b\left(\rho_{i+1 / 2}^{n}, q_{i+1 / 2}^{n}\right)\right] q_{i+1 / 2}^{n+1}=\tilde{q}_{i+1 / 2}^{n+1}-r_{n} h a_{i+1 / 2} \rho_{i+1 / 2}^{n+1} .
$$

In (5.7) $a_{i+1 / 2}$ is $a\left(\left(i+\frac{1}{2}\right) h\right), \tilde{q}_{i+1 / 2}^{n+1}$ the value computed in (3.9) and $\rho_{i+1 / 2}^{n+1}$ in (3.8). The treatment of the boundary conditions is the same.

Now for the Lax-Friedrichs scheme or for the Glimm scheme, we denote by $q_{i}^{n+1}$ the value computed in (4.4), or in (4.10), and replace it with

$$
\left[1+r_{n} h b\left(\rho_{i}^{n}, q_{i}^{n}\right)\right] q_{i}^{n+1}=\tilde{q}_{i}^{n+1}-r_{n} h a(i h) \rho_{i}^{n+1},
$$

without any change on the boundaries. We have the following result.

THEOREM 4. With the same hypotheses as in Theorem 2 and in Theorem 3, respectively, and provided that $P_{1}(\rho)$ tends to infinity with $\rho$, the three schemes studied above give approximate solutions belonging to the bounded convex set $K\left(R_{n}\right)$ at any time $t_{n}$ where $R_{n}$ is defined by

$$
R_{n}=R_{0}+|a|_{L^{\infty}} t_{n}
$$


The stability condition becomes

$$
r_{n} \lambda\left(R_{n}\right) \leqslant \frac{1}{2} \quad \text { (or } 1 \text { respectively), }
$$

at time $t_{n}$. The parameters $r_{n}$ may be chosen such that the increasing of the sequence $t_{n}$ behaves like $\sqrt{n h}$ if $p$ does not grow faster than a polynomial near infinity. Namely, we have for $p(\rho)=\rho^{1+2 \alpha} /(1+2 \alpha), \alpha>0$,

$$
r_{n}=\frac{1}{2}\left(\alpha R_{n}+1\right)^{-1} \text {. }
$$

Moreover, we cannot be sure to go beyond the time

$$
T=\left(P_{1}(+\infty)-R_{0}\right) /|a|_{L^{\infty}}
$$

if $P_{1}(\rho)$ is bounded on $] 1,+\infty[$.

Proof. If $\left(\rho_{i+1 / 2}^{n+1}, \tilde{q}_{i+1 / 2}^{n+1}\right)$ from (5.7) belongs to $K\left(R_{n}\right)$, and, since

$$
\left|q_{i+1 / 2}^{n+1}\right| \leqslant\left|\tilde{q}_{i+1 / 2}^{n+1}\right|+r_{n} h|a|_{L^{\infty}},
$$

it is obvious by taking $R_{n+1}=R_{n}+r_{n} h|a|_{L^{\infty}}$ instead of $R_{n}$ in (2.11) that we obtain the computed values $\left(\rho_{i+1 / 2}^{n+1}, q_{i+1 / 2}^{n+1}\right)$ lying in $K\left(R_{n+1}\right)$. The arguments apply to both the other schemes and thus we get (5.9) in each case. This sequence $R_{n}$ satisfies (5.9). To estimate $r_{n}$, we have, by using (2.14) and (2.11),

$$
\lambda\left(R_{n}\right)=\operatorname{Sup}_{(\rho, q) \in K\left(R_{n}\right)}\left[\frac{|q|}{\rho}+\sqrt{p^{\prime}(\rho)}\right]=\operatorname{Sup}_{\rho}\left\{R_{n}-P_{1}(\rho)+\sqrt{p^{\prime}(\rho)}\right\}
$$

which gives by (1.6)

$$
\lambda\left(R_{n}\right)=\sqrt{p^{\prime}\left(\rho_{*}^{n}\right)}, \quad\left(\rho_{*}^{n}=P_{1}^{-1}\left(R_{n}\right)\right)
$$

Thus (5.10) holds for

$$
r_{n}=\left(2 \sqrt{p^{\prime}\left(\rho_{*}^{n}\right)}\right)^{-1}
$$

Now, for $p^{\prime}(\rho)=\rho^{2 \alpha}$, we have $P_{1}\left(\rho_{*}^{n}\right)=\alpha^{-1}\left(\left(\rho_{*}^{n}\right)^{\alpha}-1\right)$, and thus $\alpha R_{n}+1=$ $\sqrt{p^{\prime}\left(\rho_{*}^{n}\right)}$, which gives (5.11) by using (5.13). Since $R_{n}$ is given by (5.9), we have

$$
t_{n+1}^{2}-t_{n}^{2}=\frac{\left(t_{n+1}+t_{n}\right)}{2\left(1+\alpha R_{0}+\alpha t_{n}|a|_{L^{\infty}}\right)} \geqslant \frac{h}{\alpha|a|_{L^{\infty}}+\left(\alpha R_{0}+1\right) / t_{n}} \geqslant c h,
$$

where $c$ is a constant. With the same arguments we have for some constant $c^{\prime}$

$$
t_{n+1}^{2}-t_{n}^{2} \leqslant \frac{3 h / 2}{\alpha|a|_{L^{\infty}}+\left(\alpha R_{0}+1\right) / t_{n}} \leqslant c^{\prime} h
$$

A summation on $n$ gives, from (5.15) and (5.14),

$$
\sqrt{c n h} \leqslant t_{n} \leqslant \sqrt{c^{\prime} n h} \text {. }
$$

Finally, if $P_{1}$ is bounded, we have to compute with a convex set $K\left(R_{0}+|a|_{L^{\infty}} T\right)$ which is unbounded by reaching time $T$ given in (5.12), and (3.10) does not hold any more. This completes the proof of Theorem 4.

The hypothesis on the behavior of $p$ near infinity is satisfied in all the physically meaningful applications as long as $\rho$ may become so large. In practice the growth of $R_{n}$ is not very important in the other cases. 
Note also that term $a$ may be replaced with any bounded continuous function of $(x, \rho, q)$ on $] 0,1[\times[0,+\infty[\times \mathbf{R}$ and Theorem 4 is still true.

6. Application to a Simple Supersonic Model. We consider now the equation

$$
\frac{\partial^{2} w}{\partial t^{2}}=\frac{\partial}{\partial x}\left[\frac{1}{2}\left(\frac{\partial w}{\partial x}\right)^{2}\right]
$$

which takes the form of a hyperbolic system of two equations by putting

$$
u=\frac{\partial w}{\partial x}, \quad v=\frac{\partial w}{\partial t} .
$$

We get

$$
\begin{gathered}
\frac{\partial u}{\partial t}=\frac{\partial v}{\partial x}, \\
\frac{\partial v}{\partial t}=\frac{\partial}{\partial x}\left(\frac{u^{2}}{2}\right) .
\end{gathered}
$$

This system is hyperbolic for $u>0$ only, corresponding to a supersonic phase. Note that we may consider $t$ as a time variable though this model involves effectively a stationary two-dimensional problem, i.e., $x$ and $t$ are both space variables. The variables $u$ and $v$ correspond to the components of $a$.

For that system we try to solve a Riemann problem near the sonic value velocity $u=0$ and find a discontinuity on $v$ with respect to $u$ though $u$ is still continuous. Thus (6.4) is satisfied but not (6.3). As a matter of fact a Dirac mass appears along the characteristic line $u=0$, and we shall get rid of it by multiplying (6.3) by $u$ and (6.4) by $v$ and sum them. A new conservative form appears, involving the variables $\rho=u$ and $q=u v$, which is rather near the system (1.4), (1.5).

This Riemann problem is the following: solve (6.3), (6.4) with the data

$$
(u(x, 0), v(x, 0))= \begin{cases}(1, a) & \text { for } x<0 \\ (1,-a) & \text { for } x>0\end{cases}
$$

where $a$ is a constant to be defined later. We find a solution given by

$$
u(x, t)=\operatorname{Min}\left(\frac{x^{2}}{t^{2}}, 1\right), \quad v(x, t)= \begin{cases}a & \text { for } x<-t, \\ a+\frac{2}{3}\left(\frac{|x|^{3}}{t^{3}}-1\right) & \text { for }-t<x<0 \\ -v(-x, t) & \text { for } x>0 .\end{cases}
$$

Obviously $u$ is a continuous function but for $a>\frac{2}{3} v$ has a jump at $x=0$ (corresponding to $u=0$ ).

We now write the $(\rho, q)$-system; we get

$$
\begin{gathered}
\frac{\partial \rho}{\partial t}-\frac{\partial}{\partial x}\left(\frac{q}{\rho}\right)=0, \\
\frac{\partial q}{\partial t}-\frac{\partial}{\partial x}\left(\frac{\rho^{3}}{3}+\frac{q^{2}}{2 \rho^{2}}\right)=0 .
\end{gathered}
$$

The Riemann invariants for this system are given, respectively, by

$$
v=v_{0} \pm \frac{2}{3} u^{3 / 2} \quad\left(q=\rho\left(v_{0} \pm \frac{2}{3} \rho^{3 / 2}\right)\right),
$$


for $v_{0}$ constant. Expressed in terms of $(u, v)$ such invariants can cross the axe $u=0$ in the plane $(u, v)$ at any point $(0, v)$ with $v \neq 0$. This is producing some discontinuity on $v$. Moreover, for the $(\rho, q)$-model, the point $(0,0)$ only belongs to the intersection of the axe $\{\rho=0\}$ with any Riemann invariant, hiding all discontinuity on $v$ since $q$ is still continuous. As a matter of fact $q$ is given by

$$
q(x, t)=\frac{x|x|}{t}\left[a+\frac{2}{3}\left(\frac{|x|^{3}}{t^{3}}-1\right)\right] \text { for }|x|<t
$$

in the previous example. Moreover, the convexity of the Riemann invariants is not changed. A set limited by them exists in both cases and is bounded and convex. Then all the results of Sections 2, 3, 4, and 5 apply here. We can generalize it for

$$
\frac{\partial^{2} w}{\partial t^{2}}=\frac{\partial}{\partial x}\left\{f\left(\frac{\partial w}{\partial x}\right)\right\}
$$

with $f \in C^{2}(\mathbf{R})$, decreasing on $]-\infty$, 0 , increasing and convex on $] 0,+\infty[$. We also write $\rho=\partial w / \partial x$ and $q=\rho \partial w / \partial t$ to obtain the system

$$
\begin{gathered}
\frac{\partial \rho}{\partial t}-\frac{\partial}{\partial x}\left(\frac{q}{\rho}\right)=0 \\
\frac{\partial q}{\partial t}-\frac{\partial}{\partial x}\left\{F(\rho)+\frac{q^{2}}{2 \rho^{2}}\right\}=0
\end{gathered}
$$

with $F(\rho)=\int_{0}^{\rho} y f^{\prime}(y) d y$.

This system is of hyperbolic type for $\rho>0$. The Riemann invariants are given by

$$
q=\rho\left(v_{0} \pm F_{0}(\rho)\right),
$$

in which

$$
F_{0}(\rho)=\int_{0}^{\rho} \sqrt{f^{\prime}(y)} d y .
$$

The convexity property is true if $f$ satisfies

$$
\forall \rho>0, \quad 4 f^{\prime}(\rho)+\rho f^{\prime \prime}(\rho) \geqslant 0,
$$

obtained by derivating twice $q$ in (6.14). This condition is similar to (1.6) and the application of the results of Sections 2, 3, 4, and 5 are still suitable here. We find that the values of the approximate solution always lie in the set

$$
K\left(R_{0}\right)=\left\{(\rho, q) ; \rho \geqslant 0,|q|+\rho F_{0}(\rho)-\rho R_{0} \leqslant 0\right\},
$$

if $R_{0}$ is such that the initial data belongs to $K\left(R_{0}\right)$, that is

$$
R_{0}=\operatorname{Sup}_{x \in] 0,1[}\left\{\left|v_{0}(x)\right|+F_{0}\left(u_{0}(x)\right)\right\}
$$

for $v_{0} \in L^{\infty}(] 0,1[), u_{0} \in L^{\infty}(] 0,1[)$ nonnegative. The boundary conditions are $v=0$ (or $q=0$ ) as above. Since the speed of the waves is estimated by

$$
\lambda\left(R_{0}\right)=\operatorname{Sup}_{(\rho, q) \in K\left(R_{0}\right)}\left\{\sqrt{f^{\prime}(\rho)}\right\}=F_{0}^{-1}\left(R_{0}\right),
$$

the stability conditions are to be written as

$$
r \lambda\left(R_{0}\right)=r F_{0}^{-1}\left(R_{0}\right)<\frac{1}{2} \quad(\text { or } 1) .
$$

The image of $K\left(R_{0}\right)$ in the $(u, v)$-plane is also a bounded convex set if $f$ is convex on $] 0,+\infty[$, but it contains other points $(0, v)$ than $(0,0)$. This may be a source of 
trouble, as above for $f(u)=u^{2} / 2$. Nevertheless, the numerical schemes studied here are applicable and stable in this set, as shown in [6], [10]. The invariance of such sets was stated in [1], from another point of view than the numerical one. See also [12], [14]. Other generalizations of the upwind scheme are studied in [13]. We also recall that (6.16) may involve the stability in a bounded convex set in which $\lambda\left(R_{0}\right)$ defined by (6.19) is unbounded, as for $f(\rho)=-\rho^{-\gamma}$ with $2-\sqrt{3}<\gamma$ $<2+\sqrt{3}$. Such an example is given by the isentropic gas dynamics equations in Lagrangian coordinates, as seen at the end of Section 1.

The author wants to thank Professor P. A. Raviart who gave him the idea of breaking some well-known schemes to two steps in order to study them as transport-projection methods. This paper generalizes [8], [9], the results of which were found for an oceanic model with obstacles near the surface. This research was supported by C.N.E.X.O. under contract No. 80.2212 .

Laboratoire d'Analyse Numérique

I.N.S.A. 20, Avenue des Buttes de Coësmes

35043 Rennes Cedex, France

1. K. N. Chueh, C. C. Conley \& J. A. Smoller, "Positively invariant regions for systems of nonlinear diffusion equations," Indiana Univ. Math. J., v. 26, 1977, pp. 379-392.

2. J. Glimm, "Solutions in the large for nonlinear hyperbolic systems," Comm. Pure Appl. Math., v. 18, 1965, pp. 697-715.

3. S. K. Godunov, "A difference method for numerical calculation of discontinuous equations of hydrodynamics," Mat. Sb., v. 47 (89), 1959, pp. 271-300.

4. P. D. LAX, "Hyperbolic systems of conservation laws. II," Comm. Pure Appl. Math., v. 10, 1957, pp. 537-566.

5. P. D. LAX, "Shock waves and entropy," Contribution to Nonlinear Functional Analysis (E. H. Zarantonello, Ed.), Academic Press, New York, 1971.

6. A. Y. Le Roux, Approximation de Quelques Problèmes Hyperboliques Non Linéaires, Thèse d'Etat, Rennes, 1979.

7. A. Y. LE Roux, "Etude du problème mixte pour une équation quasilinéaire du premier ordre," C. R. Acad. Sci. Paris Ser. A-B, v. 285, 1977, pp. 351-354.

8. A. Y. Le Roux, Stabilité de Schémas Numériques Adaptés à Certains Modèles Océaniques Non Linéaires, Rapport C.N.E.X.O. 78, 1963 (1980).

9. A. Y. Le Roux, "Stabilité numérique demodèles océaniques non linéaires," C. R. Acad. Sci. Paris Ser. A-B., v. 290, 1980, pp. 885-888.

10. A. Y. LE Roux, "Stability of numerical schemes solving quasi-linear wave equations," Math. Comp., v. 36, 1981, pp. 93-105.

11. T. P. LIU, "Uniqueness of weak solutions of the Cauchy problem for general $2 \times 2$ conservation laws,” J. Differential Equations, v. 20, 1976, pp. 369-388.

12. T. Nishida \& J. A. Smoller, "Mixed problem for nonlinear conservation laws," J. Differential Equations, v. 23, 1977, pp. 244-269.

13. S. OsHER, "Numerical solution of singular perturbation problems and hyperbolic systems of conservation laws," in Analytical and Numerical Approaches to Asymptotic Problems in Analysis, Math. Studies \#47 (O. Axelsson, L. S. Franc, A. van der Sluis, Eds.), North-Holland, Amsterdam, 1981, pp. 179-204.

14. B. WENDROFF, "The Riemann problem for materials with non convex equation of state $I$. Isentropic flow," J. Math. Pures Appl., v. 38, 1977, pp. 454-466; II. General flow, J. Math. Pures Appl., v. 38,1977 , pp. $640-658$. 